\title{
The Continuous Classical Optimal Boundary Control of a Couple Linear Elliptic Partial Differential Equations
}

\author{
Jamil A. Ali Al-Hawasy and Safaa J. Mohammed Al-Qaisi \\ Department of Mathematics, College of Science, Al-Mustansiriyah University, Baghdad-Iraq, \\ Corresponding author: jhawassy17@uomustansiriyah.edu.iq, hawasy20@yahoo.com
}

\begin{abstract}
This paper is concerned with the proof of the existence and uniqueness theorem for the solution of the state vector of a couple linear elliptic partial differential equations using the Galerkin method, where the continuous classical boundary control vector is given. Also, the existence theorem of a continuous classical boundary optimal control vector governed by the couple of linear elliptic partial differential equation is proved. The existence and the uniqueness solution of the couple of adjoint equations associated with the considered couple of the state equations studied. The derivation of the Fréchet derivative of the Hamiltonian is developed. The necessary conditions theorem of optimality of this problem is proved. [DOI: 10.22401/ANJS.00.1.18]
\end{abstract}

Keywords: Classical optimal boundary control, couple of linear elliptic partial differential equations, necessary conditions.

\section{Introduction}

The optimal control problems play an important role in many fields of real life problems, for examples in robotics [Braun \& Vijayakumar 2013], in an electric power [Wang \& Lin 2013], in civil engineering [Amini \& Afshar 2008], in Aeronautics and Astronautics [Lessard \& Lall 2012], in medicine [Liddo 2016], in economic [Derakhshan 2015], in heat conduction [Yilmaz \& Mahariq 2016], in biology [Chalak 2014] and many others fields.

The importance of optimal control problems encouraged many researchers interested to study the optimal control problems for systems which are governed either by nonlinear ordinary differential equations as in [Warga 1972] and [Orpel 2009] or by linear partial differential equations as in [Lions 1972] or are governed by nonlinear partial differential equations either of a hyperbolic type as in [Al-Hawasy 2008] or of a parabolic type as in [Chryssoverghi \& Al-Hawasy 2010] or by an elliptic type as in [Bors \& Walczak 2005], or optimal control problem are governed by a couple of linear partial differential equations of a hyperbolic type as in [Al-Hawasy 2016] or of a parabolic type as in [Kadhem 2015] or by an elliptic type as in [Al-Rawdhanee 2015], or of an elliptic type but involve a boundary control as in [Vexler 2007]. While the optimal control problem which is considered in this work is optimal boundary (Neumann boundary) control problem governed by a couple of linear partial differential equations of elliptic type. The control is represented here, by a control vector while the state is represented state.

In this paper, the existence theorem of a uniqueness state vector solution of a couple linear elliptic partial differential equations where the continuous classical boundary control vector is given is proved at first using the Galerkin method. Second the existence theorem of a continuous classical boundary optimal control vector governed by the couple of linear partial differential equation of elliptic type is proved. The existence theorem of a uniqueness solution of the couple of adjoint vector equations associated with the couple of state equations is studied. The derivation of the Fréchet derivative of the Hamiltonian is developed. Finally the theorem of necessary conditions for optimality of the considered problem is proved.

\section{Description of the Problem}

Let $\Omega \subset R^{2}$ be an open and bounded domain with Lipschitz boundary $\Gamma=\partial \Omega$. Consider the continuous classical optimal boundary control consisting of a couple linear elliptic state equation with Neumann boundary conditions.

$A_{1} y_{1}+a_{0}(x) y_{1}-b(x) y_{2}=f_{1}(x)$, in $\Omega \ldots$ (1)

$A_{2} y_{2}+b_{0}(x) y_{2}+b(x) y_{1}=f_{2}(x)$, in $\Omega$... (2)

$\sum_{i, j=1}^{n} a_{i j} \frac{\partial y_{1}}{\partial n}=u_{1}$, on $\Gamma$ 
$\sum_{i, j=1}^{n} b i j \frac{\partial y_{2}}{\partial n}=u_{2}$, on $\Gamma$

with

$A_{1} y_{1}=-\sum_{i, j=1}^{n} \frac{\partial}{\partial x j}\left(a_{i j}(x) \frac{\partial y_{1}}{\partial x i}\right)$,

$A_{2} y_{2}=-\sum_{i, j=1}^{n} \frac{\partial}{\partial x j}\left(b_{i j}(x) \frac{\partial y_{2}}{\partial x i}\right)$

where $\quad a_{0}(x), b_{0}(x), b(x), a_{i j}(x), b_{i j}(x) \in$ $C^{\infty}(\Omega), \quad$ and $\quad\left(u_{1}, u_{2}\right)=\left(u_{1}(x), u_{2}(x)\right) \in$ $\left(L^{2}(\Gamma)\right)^{2}$ is the classical boundary control vector, $\left(y_{1}, y_{2}\right)=\left(y_{1}(x), y_{2}(x)\right) \in\left(H^{1}(\Omega)\right)^{2}$

is the state vector, corresponding to the control vector, and $\left(f_{1}, f_{2}\right)=\left(f_{1}(x), f_{2}(x)\right) \in$ $\left(L^{2}(\Omega)\right)^{2}$ is a vector of a given function, for all $x \in \Omega$.

The set of admissible control $\vec{W} \subset\left(L^{2}(\Gamma)\right)^{2}$ is $\vec{W}=\left\{\left(u_{1}, u_{2}\right) \in\left(L^{2}(\Gamma)\right)^{2} \mid\left(u_{1}, u_{2}\right) \in U_{1} \times\right.$

$U_{2}=\vec{U} \subset R^{2}$ a. e. in $\left.\Gamma\right\}$

where $U_{1}$ and $U_{2}$ are convex sets.

The cost functional is

$$
\begin{aligned}
& \underset{\vec{u}}{\operatorname{Min}} G_{0}(\vec{u})=\frac{1}{2}\left\|y_{1}-y_{1 d}\right\|_{L^{2}(\Omega)}^{2}+ \\
& \frac{1}{2}\left\|y_{2}-y_{2 d}\right\|_{L^{2}(\Omega)}^{2}+\frac{1}{2}\left\|u_{1}\right\|_{L^{2}(\Gamma)}^{2}+\frac{1}{2}\left\|u_{2}\right\|_{L^{2}(\Gamma)}^{2}
\end{aligned}
$$

The continuous classical optimal boundary control problem is, minimize the cost functional (6), subject to the condition $\vec{u}=\left(u_{1}, u_{2}\right) \in \vec{W}$.

Let $\quad \vec{V}=V \times V=H^{1}(\Omega) \times H^{1}(\Omega)$. We denote $(v, v)_{\Omega}\left((v, v)_{\Gamma}\right)$ and $\|v\|_{L^{2}(\Omega)}\left(\|v\|_{L^{2}(\Gamma)}\right)$ as the inner product and the norm in $L^{2}(\Omega)\left(L^{2}(\Gamma)\right)$, by $(v, v),\|v\|_{H^{1}(\Omega)}$ the inner product and the norm in $H^{1}(\Omega)$, by $(\vec{v}, \vec{v})_{\Omega}=$ $\sum_{i=1}^{2}\left(v_{i}, v_{i}\right)$ and $\|\vec{v}\|_{\left(L^{2}(\Omega)\right)^{2}}=\sum_{i=1}^{2}\left\|v_{i}\right\|_{L^{2}(\Omega)}$ the inner product and the norm in $L^{2}(\Omega) \times$ $L^{2}(\Omega), \quad$ by $(\vec{v}, \vec{v})=\sum_{i=1}^{2}\left(v_{i}, v_{i}\right) \quad$ and $\|\vec{v}\|_{\left(H^{1}(\Omega)\right)^{2}}=\sum_{i=1}^{2}\left\|v_{i}\right\|_{H^{1}(\Omega)}$ the inner product and the norm in $\vec{V}$ and $\vec{V}^{*}$ is the dual of $\vec{V}$.

\section{Weak Formulation of the State Equations}

The weak form of problem (1-4) are obtained by multiplying both sides of (1-2) by $v_{1} \in V$ and $v_{2} \in V$, respectively, integrating both sides and then using the generalized Green's theorem (in Hilbert Space) for the terms which have the $2^{\text {nd }}$ order derivatives, once get:

$a_{1}\left(y_{1}, v_{1}\right)+\left(a_{0} y_{1}, v_{1}\right)_{\Omega}-\left(b y_{2}, v_{1}\right)_{\Omega}=$ $\left(f_{1}, v_{1}\right)_{\Omega}+\left(u_{1}, v_{1}\right)_{\Gamma}, \forall v_{1} \in V$ $a_{2}\left(y_{2}, v_{2}\right)+\left(b_{0} y_{2}, v_{2}\right)_{\Omega}+\left(b y_{1}, v_{2}\right)_{\Omega}=$

$\left(f_{2}, v_{2}\right)_{\Omega}+\left(u_{2}, v_{2}\right)_{\Gamma}, \forall v_{2} \in V$

Adding (7) and (8), to get:

$a(\vec{y}, \vec{v})=F(\vec{v}), \forall \vec{v} \in \vec{V}$

where $a(\vec{y}, \vec{v})=a_{1}\left(y_{1}, v_{1}\right)+\left(a_{0} y_{1}, v_{1}\right)_{\Omega}-$

$\left(b y_{2}, v_{1}\right)_{\Omega}+a_{2}\left(y_{2}, v_{2}\right)+\left(b_{0} y_{2}, v_{2}\right)_{\Omega}+$

$\left(b y_{1}, v_{2}\right)_{\Omega}$

with:

$a_{1}\left(y_{1}, v_{1}\right)=\sum_{i, j=1}^{n} a_{i j} \frac{\partial y_{1}}{\partial x i} \cdot \frac{\partial v_{1}}{\partial x j}$

$a_{2}\left(y_{2}, v_{2}\right)=\sum_{i, j=1}^{n} b_{i j} \frac{\partial y_{2}}{\partial x i} \cdot \frac{\partial v_{2}}{\partial x j}$,

$a_{i}\left(y_{i}, y_{i}\right) \geq c_{i}\left\|y_{i}\right\|_{H^{1}(\Omega)}^{2}$ where $c_{i}>0, \mathrm{i}=1,2$

$\left|a_{i}\left(y_{i}, v_{i}\right)\right| \leq \bar{c}_{i}\left\|y_{i}\right\|_{H^{1}(\Omega)}\left\|v_{i}\right\|_{H^{1}(\Omega)}$

where $\bar{c}_{i}>0, \mathrm{i}=1,2$ and

$F(\vec{v})=\left(f_{1}, v_{1}\right)_{\Omega}+\left(u_{1}, v_{1}\right)_{\Gamma}+\left(f_{2}, v_{2}\right)_{\Omega}+$

$\left(u_{2}, v_{2}\right)_{\Gamma}$

The following assumptions are useful for many employments in this work.

\section{Assumptions (A):}

a $-a(\vec{y}, \vec{v})$ is coercive, i.e., $a(\vec{y}, \vec{y}) \geq$ $c\|\vec{y}\|_{\left(H^{1}(\Omega)\right)^{2}}^{2}$.

b $-|a(\vec{y}, \vec{v})| \leq c_{1}\|\vec{y}\|_{\left(H^{1}(\Omega)\right)^{2}}\|\vec{v}\|_{\left(H^{1}(\Omega)\right)^{2}}$, where $c_{1}>0$.

$\mathrm{c}-|F(\vec{v})| \leq c_{2}\|\vec{v}\|_{\left(H^{1}(\Omega)\right)^{2}}, \forall \vec{v} \in \vec{V}, c_{2}>0$.

To find the solution of problem (9), the Galerkin's method is used by choosing an approximation subspace $\vec{V}_{n} \subset \vec{V}\left(\vec{V}_{n}\right.$ be the set of continuous and piecewise affine function in $\Omega$ ), hence problem (9) will be reduced to the approximation problem.

$a\left(\vec{y}_{n}, \vec{v}\right)=F(\vec{v}), \forall \vec{y}_{n}, \vec{v} \in \vec{V}_{n}$

\section{Theorem 3.1:}

For any fixed (given) control $\vec{u} \in$ $\left(L^{2}(\Gamma)\right)^{2}$, problem (9) has a unique approximation solution $\vec{y}_{n} \in \vec{V}_{n}$.

Proof: Let $\left\{\vec{\varphi}_{1}, \vec{\varphi}_{2}, \cdots, \vec{\varphi}_{n}\right\}$ be a basis of $\vec{V}_{n}$, then the approximation solution is:

$\vec{y}_{n}=\sum_{i=1}^{n} c_{j} \vec{\varphi}_{j}\left(x_{1}, x_{2}\right)$

where $\vec{\varphi}_{j}=\left((2-l) \varphi_{k},(l-1) \varphi_{k}\right), l=1,2$, $j=k+n(l+1)$ and $c_{j}=c_{l k}$ is a unknown constant, $\forall j=1,2, \cdots, n$, with $n=2 N$.

By substituting (12) in (11), with $\vec{v}=\vec{\varphi}_{i}$, to get:

$\sum_{j=1}^{n} c_{i} a\left(\vec{\varphi}_{j}, \vec{\varphi}_{i}\right)=F\left(\vec{\varphi}_{i}\right), i=1,2, \cdots, n$

which is equivalent to the following linear algebraic system.

$A_{n \times n} C_{n \times 1}=b_{n \times 1}$ 
Easily one can get that through using assumption (A-a), problem (14) has a unique solution which gives the existence of a unique solution of (11).

\section{Remark 3.1, [6]:}

For each $\vec{v} \in \vec{V}$, there exists a sequence $\left\{\vec{\varphi}_{n}\right\}$ with $\vec{\varphi}_{n} \in \vec{V}_{n}, \forall n$ such that $\vec{\varphi}_{n} \rightarrow \vec{v}$ strongly in $\vec{V}$.

Now, from this remark and the weak form (11), we get that there exists a sequence of weak forms

$a\left(\vec{y}_{n}, \vec{\varphi}_{n}\right)=F\left(\vec{\varphi}_{n}\right), \forall \vec{y}_{n}, \vec{\varphi}_{n} \in \vec{V}_{n}, \forall n$

which has a sequence of solutions $\left\{\vec{y}_{n}\right\}_{n=1}^{\infty}$.

\section{Theorem 3.2:}

The sequence of solutions $\left\{\vec{y}_{n}\right\}_{n=1}^{\infty}$ converges strongly to $\vec{y}$.

Proof:

Since $\vec{y}_{n}$ is a solution of (15), then from assumptions (A- a \& c) we get

$\left\|\vec{y}_{n}\right\|_{\left(H^{1}(\Omega)\right)^{2}} \leq c_{2}$, where $c_{2}>0, \forall n$

By Alaoglu theorem, there exists a subsequence of $\left\{\vec{y}_{n}\right\}$ say a gain $\left\{\vec{y}_{n}\right\}$ such that $\vec{y}_{n} \rightarrow \vec{y}$ weakly in $\vec{V}$. To prove, that the sequence of solution $\left\{\vec{y}_{n}\right\}_{n=1}^{\infty}$ of problem (15) converges to the solution of problem (9).

First, from the above steps and since $\vec{\varphi}_{n} \rightarrow \vec{v}$ strongly in $\vec{V}$, yields

$\left|a\left(\vec{y}_{n}, \vec{\varphi}_{n}\right)-a(\vec{y}, \vec{v})\right| \leq\left|a\left(\vec{y}_{n}, \vec{\varphi}_{n}-\vec{v}\right)\right|+$ $\left|a\left(\vec{y}_{n}-\vec{y}, \vec{v}\right)\right|$

$\leq c_{1}\left\|\vec{y}_{n}\right\|_{\left(H^{1}(\Omega)\right)^{2}}\left\|\vec{\varphi}_{n}-\vec{v}\right\|_{\left(H^{1}(\Omega)\right)^{2}}+$

$\left\|\vec{y}_{n}-\vec{y}\right\|_{\left(H^{1}(\Omega)\right)^{2}}\|\vec{v}\|_{\left(H^{1}(\Omega)\right)^{2}} \longrightarrow 0$

Hence:

$a\left(\vec{y}_{n}, \vec{\varphi}_{n}\right) \rightarrow a(\vec{y}, \vec{v})$

On the other hand, from remark 3.1, $\vec{\varphi}_{n} \rightarrow \vec{v}$ weakly in $\vec{V}$, which gives $F\left(\vec{\varphi}_{n}\right) \rightarrow$ $F(\vec{v})$. To prove $\vec{y}_{n} \rightarrow \vec{y}$ strongly in $\vec{V}$, we have for fixed $\vec{v} \in \vec{V}$

$L_{\vec{v}}(\vec{w})=a(\vec{w}, \vec{v})$ is linear w.r.t. $\vec{W}$

By using assumption (A-a) and that $\vec{y}_{n}$ is a solution of weak form (15), it follows that:

$c\left\|\vec{y}-\vec{y}_{n}\right\|_{\left(H^{1}(\Omega)\right)^{2}}^{2} \leq a\left(\vec{y}-\vec{y}_{n}, \vec{y}-\vec{y}_{n}\right)=$

$a\left(\vec{y}-\vec{y}_{n}, \vec{y}\right)-a\left(\vec{y}, \vec{y}_{n}\right)+a\left(\vec{y}_{n}, \vec{y}_{n}\right)=$

$a\left(\vec{y}-\vec{y}_{n}, \vec{y}\right)=L_{\vec{y}}\left(\vec{y}-\vec{y}_{n}\right) \rightarrow 0$

Which gives $\left\{\vec{y}_{n}\right\}$ converges strongly to $\vec{y}$ with respect to $\|\cdot\|_{\left(H^{1}(\Omega)\right)^{2}}$. The uniqueness is obtained easily through using assumption (A-a) also.

\section{Existence of Optimal Control}

In this section, the following lemmas will be useful later in the proof of the existence of optimal control theorem.

\section{Lemma 4.1:}

The operator $\vec{u} \mapsto \vec{y}_{\vec{u}}$ from $\vec{W}$ to $\left(L^{2}(\Omega)\right)^{2}$ is Lipschitz continuous, i.e., $\|\overrightarrow{\Delta y}\|_{\left(L^{2}(\Omega)\right)^{2}} \leq c_{3}\|\overrightarrow{\Delta u}\|_{\left(L^{2}(\Omega)\right)^{2}}$, with $c_{3}>0$.

\section{Proof:}

Let $\vec{u}, \vec{u}^{\prime} \in \vec{W}$ be two controls vectors of the weak form (9), $\vec{y}, \vec{y}^{\prime}$ be the corresponding state solutions vectors of these controls. Subtracting the above two obtained weak forms from (9), then setting $\overrightarrow{\Delta y}=\vec{y}^{\prime}-\vec{y}$ and $\overrightarrow{\Delta u}=\vec{u}^{\prime}-\vec{u}$, with $\vec{v}=\overrightarrow{\Delta y}$, to get

$a(\overrightarrow{\Delta y}, \overrightarrow{\Delta y})=\left(\Delta u_{1}, \Delta y_{1}\right)_{\Gamma}+\left(\Delta u_{2}, \Delta y_{2}\right)_{\Gamma}$

After taking the absolute value of its both sides, using assumption (A-a), the CauchySchwarz inequality and finally using the trace operator to get

$c\|\overrightarrow{\Delta y}\|_{\left(H^{1}(\Omega)\right)^{2}}^{2} \leq|a(\overrightarrow{\Delta y}, \overrightarrow{\Delta y})| \leq$
$c_{1}\|\overrightarrow{\Delta u}\|_{\left(L^{2}(\Gamma)\right)^{2}}\|\overrightarrow{\Delta y}\|_{\left(H^{1}(\Omega)\right)^{2}}$,

then

$\|\overrightarrow{\Delta y}\|_{\left(H^{1}(\Omega)\right)^{2}} \leq c_{2}\|\overrightarrow{\Delta u}\|_{\left(L^{2}(\Gamma)\right)^{2}}$ where $c_{2}=\frac{\mathrm{c}_{1}}{\mathrm{c}}$ which gives

$\|\overrightarrow{\Delta y}\|_{\left(L^{2}(\Omega)\right)^{2}} \leq c_{3}\|\overrightarrow{\Delta u}\|_{\left(L^{2}(\Gamma)\right)^{2}}$ where $c_{3}=b c_{2}$

Hence the operator $\vec{u} \longmapsto \vec{y}_{\vec{u}}$ is Lipschitz continuous on $\left(L^{2}(\Omega)\right)^{2}$.

\section{Lemma 4.2[3]:}

The norm $\|\cdot\|_{L^{2}(\Omega)}\left(\|\cdot\|_{L^{2}(\Gamma)}\right)$ is weakly lower semicontinuous.

\section{Lemma 4.3:}

The cost function (6) is weakly lower semicontinuous.

Proof:

From lemma 4.2, the norm $\|.\|_{L^{2}(\Gamma)}$ is weakly lower semicontinuous, on the other hand when $\vec{u}_{n} \rightarrow \vec{u}$ weakly in $\left(L^{2}(\Gamma)\right)^{2}$, then by lemma $4.1 \quad \vec{y}_{n} \rightarrow \vec{y}=\vec{y}_{\vec{u}}$ weakly in $\left(L^{2}(\Omega)\right)^{2}$, which gives again from lemma 4.2, that $\left\|\vec{y}-\vec{y}_{d}\right\|_{\left(L^{2}(\Omega)\right)^{2}}^{2}$ is weakly lower semicontinuous, i.e., $G_{0}(\vec{u})$ is weakly lower semicontinuous. 


\section{Lemma 4.4 [3]:}

The norm $\|.\|_{L^{2}(\Omega)}\left(\|.\|_{L^{2}(\Gamma)}\right)$ is strictly convex.

\section{Remark 4.1:}

Using lemma 4.4, the cost function $G_{0}(\vec{u})$ is strictly convex.

\section{Theorem 4.1:}

Assume $U_{i}, \forall i=1,2$ is convex. If the cost function (6) is coercive, then there exists a continuous classical boundary optimal control for the problem (6).

Proof:

Since $U_{i}, \forall i=1,2$ is convex, then $W_{i}(\forall i=1,2)$ is convex and then $\vec{W}$ is convex. Since $G_{0}(\vec{u}) \geq 0$, and $G_{0}(\vec{u})$ is coercive then there exists a minimization sequence $\left\{\vec{u}_{n}\right\} \in \vec{W}, \forall n$, such that:

$\lim _{n \rightarrow \infty} G_{0}\left(\vec{u}_{n}\right)=\inf _{\vec{w} \in \vec{W}} G_{0}(\vec{w})$

Therefore:

$\left\|\vec{u}_{n}\right\|_{\left(L^{2}(\Gamma)\right)^{2}} \leq c, \forall n, c>0$

Then by Alaoglu theorem, there exists a subsequence of $\left\{\vec{u}_{n}\right\}$ say a gain $\left\{\vec{u}_{n}\right\}$ such that $\vec{u}_{n} \rightarrow \vec{u}$ weakly in $\left(L^{2}(\Gamma)\right)^{2}$. By theorem 3.1 , $\left\{\vec{y}_{n}\right\}$ be a sequence of solutions of a sequence of problems like (9). To prove $\left\{\vec{y}_{n}\right\}, \forall n$, is bounded in $\vec{V}$, once can used assumptions (Aa \& c), Cauchy-Schwarz inequality and the trace operator, to get:

$c\left\|\vec{y}_{n}\right\|_{\left(H^{1}(\Omega)\right)^{2}}^{2} \leq a\left(\vec{y}_{n}, \vec{y}_{n}\right)=\left|F\left(\vec{y}_{n}\right)\right|$

$\leq\left\|f_{1}\right\|_{L^{2}(\Omega)}\left\|y_{1 n}\right\|_{L^{2}(\Omega)}+\left\|u_{1 n}\right\|_{L^{2}(\Gamma)}\left\|y_{1 n}\right\|_{L^{2}(\Gamma)}+$ $\left\|f_{2}\right\|_{L^{2}(\Omega)}\left\|y_{2 n}\right\|_{L^{2}(\Omega)}+\left\|u_{2 n}\right\|_{L^{2}(\Gamma)}\left\|y_{2 n}\right\|_{L^{2}(\Gamma)}$ $\leq l_{1}\left\|y_{1 n}\right\|_{L^{2}(\Omega)}+c_{1}\left\|y_{1 n}\right\|_{H^{1}(\Omega)}+l_{2}\left\|y_{2 n}\right\|_{L^{2}(\Omega)}$

$$
+c_{2}\left\|y_{2 n}\right\|_{H^{1}(\Omega)}
$$$$
\leq \mathrm{s}\left\|\overrightarrow{\mathrm{y}}_{\mathrm{n}}\right\|_{\left(H^{1}(\Omega)\right)^{2}}
$$

where $s=r_{1}+r_{2}$ and $r_{1}=l_{1}+c_{1}, r_{1}=l_{2}+$ $c_{2}$,

Then

$\left\|\vec{y}_{n}\right\|_{\left(H^{1}(\Omega)\right)^{2}} \leq a$, where $a=\frac{\mathrm{s}}{\mathrm{c}}>0$.

Then by Alaoglu theorem there exists a subsequence of $\left\{\vec{y}_{n}\right\}$ say again $\left\{\vec{y}_{n}\right\}$ such that $\vec{y}_{n} \rightarrow \vec{y}$ weakly in $\vec{V}$ sSince for each $n, \vec{y}_{n}$ satisfies the weak form (9), then

$a\left(\vec{y}_{n}, \vec{v}\right)=F_{n}(\vec{v})=\left(f_{1}, v_{1}\right)_{\Omega}+\left(u_{1 n}, v_{1}\right)_{\Gamma}+$

$\left(f_{2}, v_{2}\right)_{\Omega}+\left(u_{2 n}, v_{2}\right)_{\Gamma}$

To show that (21) converges to

$a(\vec{y}, \vec{v})=F(\vec{v})$
First, since $\forall i, y_{\text {in }} \rightarrow y_{i}$ weakly in $V$, i.e., $y_{\text {in }} \rightarrow y_{i}$ weakly in $L^{2}(\Omega)$. Then by using the Cauchy-Schwarz inequality, one gets:

$\mid a_{1}\left(y_{1 n}, v_{1}\right)+\left(a_{0} y_{1 n}, v_{1}\right)_{\Omega}-\left(b y_{2 n}, v_{1}\right)_{\Omega}+$ $a_{2}\left(y_{2 n}, v_{2}\right)$

$+\left(b_{0} y_{2 n}, v_{2}\right)_{\Omega}+\left(b y_{1 n}, v_{2}\right)_{\Omega}-a_{1}\left(y_{1}, v_{1}\right)-$ $\left(a_{0} y_{1}, v_{1}\right)_{\Omega}$

$$
+\left(b y_{2}, v_{1}\right)_{\Omega}-a_{2}\left(y_{2}, v_{2}\right)-\left(b_{0} y_{2}, v_{2}\right)_{\Omega}
$$$$
-\left(b y_{1}, v_{2}\right)_{\Omega} \mid
$$

$\leq c_{1}\left\|y_{1 n}-y_{1}\right\|_{H^{1}(\Omega)}\left\|v_{1}\right\|_{H^{1}(\Omega)}$ $+c_{2}\left\|y_{1 n}-y_{1}\right\|_{L^{2}(\Omega)}\left\|v_{1}\right\|_{L^{2}(\Omega)}$

$+c_{3}\left\|y_{2 n}-y_{2}\right\|_{L^{2}(\Omega)}\left\|v_{1}\right\|_{L^{2}(\Omega)}+$

$c_{4}\left\|y_{2 n}-y_{2}\right\|_{H^{1}(\Omega)}\left\|v_{2}\right\|_{H^{1}(\Omega)}+c_{5} \| y_{2 n}-$

$y_{2}\left\|_{L^{2}(\Omega)}\right\| v_{2} \|_{L^{2}(\Omega)}+$

$c_{6}\left\|y_{1 n}-y_{1}\right\|_{L^{2}(\Omega)}\left\|v_{2}\right\|_{L^{2}(\Omega)} \rightarrow 0$

Second, and on the other hand, since $\vec{u}_{n} \rightarrow \vec{u}$ weakly in $\left(L^{2}(\Gamma)\right)^{2}$, then the right hand side of (21) converges to the right hand side of (22). Thus equation (21) converges to equation (22).

Since $G_{0}(\vec{u})$ is weakly lower semicontinuous, and $\vec{u}_{n} \rightarrow \vec{u}$ weakly in $\left(L^{2}(\Gamma)\right)^{2}$, then $G_{0}(\vec{u}) \leq \lim _{\mathrm{n} \rightarrow \infty} G_{0}\left(\vec{u}_{n}\right)=\inf _{\overrightarrow{\mathrm{w}} \in \overrightarrow{\mathrm{W}}} G_{0}(\vec{w})$, which gives

$G_{0}(\vec{u})=\inf _{\vec{w} \in \vec{W}} G_{0}(\vec{w})$

i.e., $\vec{u}$ a continuous classical optimal control. The uniqueness of $\vec{u}$ is obtained from remark 4.1 .

\section{Necessary Condition for Optimality}

The necessary condition for continuous classical optimal control is studied through the following theorem.

\section{Theorem 5.1:}

Consider the cost function (6), and the adjoint $\left(z_{1}, z_{2}\right)=\left(z_{1 u_{1}}, z_{2 u_{2}}\right)$ equations of the state equations (1-4) are given by

$A_{1} z_{1}+a_{0}(x) z_{1}+b(x) z_{2}=\left(y_{1}-y_{1 d}\right)$, in $\Omega$

$A_{2} z_{2}+b_{0}(x) z_{2}-b(x) z_{1}=\left(y_{2}-y_{2 d}\right)$, in $\Omega$

$\frac{\partial z_{1}}{\partial n}=0$, in $\Gamma$

$\frac{\partial z_{2}}{\partial n}=0$, in $\Gamma$

Then the Fréchet derivative of $G_{0}$ is given by $\left(G_{0}^{\prime}(\vec{u}), \overrightarrow{\Delta u}\right)=(\vec{z}+\vec{u}, \overrightarrow{\Delta u})$. 
Proof:

Writing the couple of the adjoint equations (23-26) by their weak forms, adding those weak forms, then substituting $\vec{v}=\overrightarrow{\Delta y}$ in the obtained equation to get, the following weak form which has a unique solution $\vec{z}=\vec{z}_{\vec{u}}$ for a given control $\vec{u} \in \vec{W}$ (the proof is similar to the proof of theorem 3.1):

$$
\begin{aligned}
& a_{1}\left(z_{1}, \Delta y_{1}\right)+\left(a_{0} z_{1}, \Delta y_{1}\right)_{\Omega}+\left(b z_{2}, \Delta y_{1}\right)_{\Omega}+ \\
& a_{2}\left(z_{2}, \Delta y_{2}\right)+\left(b_{0} z_{2}, \Delta y_{2}\right)_{\Omega} \\
& -\left(b z_{1}, \Delta y_{2}\right)_{\Omega}=\left(y_{1}-y_{1 d}, \Delta y_{1}\right)_{\Omega}+ \\
& \left(y_{2}-y_{2 d}, \Delta y_{2}\right)_{\Omega}
\end{aligned}
$$

Substituting in (7) once the solution $y_{1}$ and once again the solution $y_{1}+\Delta y_{1}$, subtracting the obtained equations one from the other, finally substituting $v_{1}=z_{1}$, to get

$\left(b \Delta y_{2}, z_{1}\right)_{\Omega}+\left(b z_{2}, \Delta y_{1}\right)_{\Omega}=-\left(\Delta u_{1}, z_{1}\right)_{\Gamma}+$

$\left(y_{1}-y_{1 d}, \Delta y_{1}\right)_{\Omega}$

Same steps can be used in equation (8) for the solutions $y_{2}$ and $y_{2}+\Delta y_{2}$ with $v_{2}=z_{2}$, to obtain

$-\left(b \Delta y_{1}, z_{2}\right)_{\Omega}-\left(b z_{1}, \Delta y_{2}\right)_{\Omega}=-\left(\Delta u_{2}, z_{2}\right)_{\Gamma}+$

$\left(y_{2}-y_{2 d}, \Delta y_{2}\right)_{\Omega}$

Adding (28) and (29), then subtracting the obtained equation from (27), to get

$\left(\Delta u_{1}, z_{1}\right)_{\Gamma}+\left(\Delta u_{2}, z_{2}\right)_{\Gamma}=\left(y_{1}-y_{1 d}, \Delta y_{1}\right)_{\Omega}+$

$\left(y_{2}-y_{2 d}, \Delta y_{2}\right)_{\Omega}$

Now, for the cost function, we have

$G_{0}(\vec{u}+\overrightarrow{\Delta u})-G_{0}(\vec{u})=\left(y_{1}-y_{1 d}, \Delta y_{1}\right)_{\Omega}+$

$\left(y_{2}-y_{2 d}, \Delta y_{2}\right)_{\Omega}+\left(u_{1}, \Delta u_{1}\right)_{\Gamma}+\left(u_{2}, \Delta u_{2}\right)_{\Gamma}+$

$\frac{1}{2}\|\overrightarrow{\Delta y}\|_{\left(L^{2}(\Omega)\right)^{2}}^{2}+\frac{1}{2}\|\overrightarrow{\Delta u}\|_{\left(L^{2}(\Gamma)\right)^{2}}^{2}$

From (30) \& (31), once get

$G_{0}(\vec{u}, \overrightarrow{\Delta u})-G_{0}(\vec{u})=(\vec{z}+\vec{u}, \overrightarrow{\Delta u})_{\Gamma}+$

$\frac{1}{2}\|\overrightarrow{\Delta y}\|_{\left(L^{2}(\Omega)\right)^{2}}^{2}+\frac{1}{2}\|\overrightarrow{\Delta u}\|_{\left(L^{2}(\Gamma)\right)^{2}}^{2}$

From lemma 4.1, it yield that

$\frac{1}{2}\|\overrightarrow{\Delta y}\|_{\left(L^{2}(\Omega)\right)^{2}}^{2}+\frac{1}{2}\|\overrightarrow{\Delta u}\|_{\left(L^{2}(\Gamma)\right)^{2}}^{2}=$

$\varepsilon(\overrightarrow{\Delta u})\|\overrightarrow{\Delta u}\|_{\left(L^{2}(\Gamma)\right)^{2}}$

where $\varepsilon(\overrightarrow{\Delta u}) \rightarrow 0$, and $\|\overrightarrow{\Delta u}\|_{\left(L^{2}(\Gamma)\right)^{2}} \rightarrow 0$ as

$\overrightarrow{\Delta u} \rightarrow 0$

Then from the Fréchet derivative of $G_{0}$, and (32-33), once get:

$\left(G_{0}^{\prime}(\vec{u}), \overrightarrow{\Delta u}\right)=(\vec{z}+\vec{u}, \overrightarrow{\Delta u})_{\Gamma}$.

\section{Theorem 5.2:}

The continuous classical optimal boundary control of (1-4) is:

$G_{0}^{\prime}(\vec{u})=\vec{z}+\vec{u}=0$ with $\vec{y}=\vec{y}_{\vec{u}}$ and $\vec{z}=\vec{z}_{\vec{u}}$.

\section{Proof:}

If $\vec{u}$ an optimal control of problem, then

$G_{0}(\vec{u})=\min _{\vec{w} \in \vec{W}} G_{0}(\vec{w}), \forall \vec{w} \in\left(L^{2}(\Gamma)\right)^{2}$

i.e., $G_{0}^{\prime}(\vec{u})=0 \Rightarrow \vec{z}=-\vec{u}$

$\overrightarrow{\Delta u}=\vec{w}-\vec{u}$

Thus necessary condition for optimality is $(\vec{z}+\vec{u}, \vec{u}) \leq(\vec{z}+\vec{u}, \vec{w}), \forall \vec{w} \in\left(L^{2}(\Gamma)\right)^{2}$.

\section{Conclusions:}

The Galerkin method is used successfully to prove the existence and the uniqueness theorem for the solution (continuous state vector) of a couple linear elliptic partial differential equations when the continuous classical boundary control vector is given. The existence theorem of a continuous classical boundary optimal control vector governing by the considered couple of linear partial differential equation of elliptic type is proved. The existence and the uniqueness solution of the couple of adjoint equations associated with the considered couple equations of the state is studied. The Fréchet derivation of the Hamiltonian is developed. The necessary conditions theorem of optimality of the considered problem is proved.

\section{References}

[1] Al-Hawasy, J. The Continuous Classical Optimal Control of a Nonlinear Hyperbolic Equation (CCOCP). Al-Mustansiriyah Journal of Science, Vol.19, No.8, pp.96110, 2008.

[2] Al-Hawasy, J. The Continuous Classical Optimal Control of a Couple Nonlinear Hyperbolic Partial Differential Equations With Equality and Inequality Constraints. Iraqi Journal of Science, Vol.57, No.2C, pp:1528-1538, 2016.

[3] Al-Hawasy, J. Optimization and Approximation for Parabolic Boundary Value Problem, Ph.D. Thesis, NTUAGreece, 2004.

[4] Al-Rawdanee, E. H. M. The Continuous Classical Optimal Control Problem of a Non-Linear Partial Differential Equations of Elliptic Type. M.Sc. Thesis, AlMustansiriyah, Baghdad-Iraq, 2015.

[5] Amini, F. and Afshar, M. Amin Modified Predictive optimal linear Control of Structures in Seismic Region. Iranian 
Journal of Science \& Technology, Transaction B, Engineering, Vol. 32, No. B2, pp 91-106, 2008.

[6] Bacopoulos, A. and Chryssoverghi, I. Numerical Solutions of Partial Differential Equations by Finite Elements Methods. Symeom Publishing Co., Athens, 2003.

[7] Bors, D. and Walczak, S. Optimal control elliptic system with distributed and boundary controls. Nonlinear Analysis 63, e1367-e1376, 2005.

[8] Braun, D. J. Petit, F. Huber, F. Haddadin, S. Albu-Schaffer, A. and Vijayakumar, S. Robots Driven by Compliant Actuators: Optimal Control Under Actuation Constraints. IEEE TRANSACTIONS ON ROBOTICS, VOL. 29, NO. 5, 2013.

[9] Chalak, M. Optimal Control for a Dispersing Biological Agent. Journal of Agricultural and Resource Economics 39(2): 271-289 ISSN 1068-5502, 2014.

[10] Chryssoverghi, I. \& Al-Hawasy, J. The Continuous Classical Optimal Control Problem of a Semi linear Parabolic Equations (CCOCP). Journal of Karbala University, Vol.8, No.3, pp.57-70, 2010.

[11] Derakhshan, M. Control Theory and Economic Policy Optimization: The Origin, Achievements and the Fading Optimism from a Historical Standpoint. International Journal of Business and Development Studies Vol. 7, No. 1, pp 529, 2015.

[12] Kadhem, G. M. The Continuous Classical Optimal Control Problem of Partial Differential Equations. M.Sc. Thesis, AlMustansiriyah, Baghdad-Iraq, 2015.

[13] Lessard, L. \& Lall, S. Optimal Controller Synthesis for the Decentralized TwoPlayer Problem with Output Feedback. American Control Conference, pp. 63146321, 2012.

[14] Liddo, A. Di. Optimal Control and Treatment of Infectious Diseases. The Case of Huge Treatment Costs. Mathematics doi:10.3390/math4020021, 2016.
[15] Lions, J. L. Optimal control of systems governed by Partial differential equations. Springer-Verlag, New York, 1972.

[16] Orpel, A. Optimal Control Problems With Higher Order Constraints. Folia Mathematica, Vol.16, No.1, pp.31-44, 2009.

[17] Vexler, B. Finite element approximation of elliptic Dirichlet optimal control problems. Numer. Funct. Anal. Optim. 28, 957-973, 2007.

[18] Wang, Y. Lin, X. Park, S. and Chang, N. Optimal Control of a Grid-Connected Hybrid Electrical Energy Storage System for Homes. 978-3-9815370-0-0/DATE13/ EDAA, 2013.

[19] Warga, J. Optimal Control of Differential and Functional Equations. Academic Press: New York and London, 1972.

[20] Yilmaz, A. Mahariq, I. and Yilmaz, F. Numerical Solutions of Optimal Control Problems for Microwave Heating. International Journal of Advances in Science Engineering and Technology, ISSN: 2321-9009 Vol.4, Issue 3, 2016. 